\title{
Optimal Constants in the Rosenthal Inequality for Random Variables with Zero Odd Moments.
}

\section{Citation}

Ibragimov, Rustam and Marat Ibragimov. 2008. Optimal constants in the Rosenthal inequality for random variables with zero odd moments. Statistics and Probability Letters 78(2): 186-189.

\section{Published Version}

http://dx.doi.org/10.1016/j.spl.2007.05.018

\section{Permanent link}

http://nrs.harvard.edu/urn-3:HUL.InstRepos:2624461

\section{Terms of Use}

This article was downloaded from Harvard University's DASH repository, and is made available under the terms and conditions applicable to Other Posted Material, as set forth at http:// nrs.harvard.edu/urn-3:HUL.InstRepos:dash.current.terms-of-use\#LAA

\section{Share Your Story}

The Harvard community has made this article openly available.

Please share how this access benefits you. Submit a story.

\section{Accessibility}




\title{
OPTIMAL CONSTANTS IN THE ROSENTHAL INEQUALITY FOR RANDOM VARIABLES WITH ZERO ODD MOMENTS ${ }^{1}$
}

\author{
Marat Ibragimov \\ Department of Probability Theory, Tashkent State Economics University
}

Rustam Ibragimov ${ }^{2}$

Department of Economics, Harvard University

Abstract. We obtain estimates for the best constant in the Rosenthal inequality $E\left|\sum_{i=1}^{n} \xi_{i}\right|^{2 m} \leq C(2 m) \max \left(\sum_{i=1}^{n} E \xi_{i}^{2 m},\left(\sum_{i=1}^{n} E \xi_{i}^{2}\right)^{m}\right)$ for independent random variables $\xi_{1}, \ldots, \xi_{n}$ with $l$ zero first odd moments, $l \geq 1$. The estimates are sharp in the extremal cases $l=1$ and $l=m$, that is, in the cases of random variables with zero mean and random variables with $m$ zero first odd moments.

Rosenthal (1970) proved the following inequality:

$$
E\left|\sum_{i=1}^{n} \xi_{i}\right|^{t} \leq C(t) \max \left(\sum_{i=1}^{n} E\left|\xi_{i}\right|^{t},\left(\sum_{i=1}^{n} E \xi_{i}^{2}\right)^{t / 2}\right)
$$

\footnotetext{
${ }^{1}$ The authors are grateful to an anonymous referee for helpful comments and suggestions.

${ }^{2}$ Corresponding author. Department of Economics, Harvard University, Littauer Center, 1805 Cambridge St., Cambridge MA 02138. Phone: (617) 496-4795. Fax: (617) 495-7730. Email: ribragim@fas.harvard.edu
} 
for all positive integers $n$ and all independent random variables (r.v.'s) $\xi_{1}, \ldots, \xi_{n}$ with $E \xi_{i}=0$, $E\left|\xi_{i}\right|^{t}<\infty, i=1, \ldots, n, t>2$, where $C(t)$ is a constant depending only on $t$. A number of papers have focused on refinements and extensions of inequality (1) and related problems (see Prokhorov, 1962; Sazonov, 1974; Nagaev and Pinelis, 1977; Pinelis, 1980, 1994; Pinelis and Utev, 1984; Johnson, Schechtman and Zinn, 1985; Utev, 1985; Hitczenko, 1990, 1994; Bestsennaya and Utev, 1991, Ibragimov and Sharakhmetov, 1995, 1997, 2001a, b; Figiel, Hitczenko, Johnson, Schechtman and Zinn, 1997; Ibragimov, 1997; de la Peña and Giné, 1999; and de la Peña, Ibragimov and Sharakhmetov, 2003). Figiel et al. (1997) and Ibragimov and Sharakhmetov $(1995,1997)$ derived the following expressions for the best constant $C_{\text {sym }}^{*}(t)$ in inequality (1) for symmetric r.v.'s: $C_{\text {sym }}^{*}(t)=1+\frac{2^{t / 2} \Gamma\left(\frac{t+1}{2}\right)}{\sqrt{\pi}}, 2<t<4, C_{\text {sym }}^{*}(t)=E\left|\theta_{1}-\theta_{2}\right|^{t}, t \geq 4$, where $\Gamma(a)=\int_{0}^{\infty} x^{a-1} e^{-x} d x$ and $\theta_{1}, \theta_{2}$ are independent Poisson r.v.'s with parameter 0.5. The proof of the expressions for $C_{\text {sym }}^{*}(t)$ in Ibragimov and Sharakhmetov $(1995,1997)$ relies on the work by Utev (1985), who obtained, among other results, sharp upper and lower bounds on $E\left|\sum_{i=1}^{n} \xi_{i}\right|^{t}$, $t \geq 4$, where $\xi_{1}, \ldots, \xi_{n}$ are independent symmetric r.v.'s with finite th moment, in terms of $\sum_{i=1}^{n} E\left|\xi_{i}\right|^{t}$ and $\left(\sum_{i=1}^{n} E \xi_{i}^{2}\right)^{t / 2}$. Bestsennaya and Utev (1991) derived a similar upper bound on even moments of sums independent mean-zero r.v.'s $\xi_{1}, \ldots, \xi_{n}$, from which the best constant in general Rosenthal's inequality (1) in the case $t=2 m$ can be deduced. Using a different proof technique, the expression for the best constant in general inequality (1) for even moments $t=2 m$ of sums of 
mean-zero r.v.'s was independently obtained in Ibragimov and Sharakhmetov (2001a). Ibragimov and Sharakhmetov (2001b) obtained the best constant in the analogue of inequality (1) for nonnegative r.v.'s. The results in Ibragimov and Sharakhmetov (1995, 1997, 2001a, b) were also presented in Ibragimov (1997). de la Peña et al. derived sharp analogues of the BurkholderRosenthal inequalities and related estimates for the expectations of functions of sums of dependent nonnegative r.v.'s and conditionally symmetric martingale differences with bounded conditional moments as well as for sums of multilinear forms.

The present paper deals with estimating the best constants in the Rosenthal's inequality for r.v.'s with $l$ zero first odd moments. Namely, let $C_{l}^{*}(t)$ denote the best constants in inequality (1) for all positive integers $n$ and all independent r.v.'s $\xi_{1}, \ldots, \xi_{n}$ with $E \xi_{i}^{2 s-1}=0$, $s=1,2, \ldots, l$. Then the following theorem holds.

Theorem 1. If $t=2 m, m \in N$, then

$$
C_{l}^{*}(2 m) \leq(2 m) ! \sum_{j=1}^{2 m} \sum_{r=1}^{j} \sum \prod_{k=1}^{r} \frac{\left(m_{k} !\right)^{-j_{k}}}{j_{k} !},
$$

where the inner sum is taken over all natural $m_{1}>m_{2}>\ldots>m_{r}$ and $j_{1}, \ldots, j_{r}$ satisfying the conditions $m_{1} j_{1}+\ldots+m_{r} j_{r}=2 m, j_{1}+\ldots+j_{r}=j, m_{i} \neq 2 s-1, i=1,2, \ldots, r, s=1,2, \ldots, l$. 
$\underline{\text { Remark 1. }}$ The value (2m)! $\sum_{j=1}^{2 m} \sum_{r=1}^{j} \sum_{k=1}^{r} \frac{\left(m_{k} !\right)^{-j_{k}}}{j_{k} !}$ in inequality (2) has a simple combinatorial sense (e.g., Sachkov, 1996): it equals the number of partitions of a set consisting of $2 m$ elements into parts the number of elements in which is not equal to $2 s-1, s=1,2, \ldots, l$.

Remark 2. As follows from the results in Pinelis and Utev (1984), Bestsennaya and Utev (1991) and Ibragimov and Sharakhmetov (1997, 2001a), bounds (2) are sharp for $l=1$ and $l=m$; in addition, when $l=m$, the right-hand side of (2) equals to the best constant $C_{\text {sym }}^{*}(2 m)$ in the Rosenthal's inequality for symmetric r.v.'s. It is also interesting to note that, in the case $l=0$, the expression on the right-hand side of (2), with the inner sum taken over all natural $m_{1}>m_{2}>\ldots>m_{r}$ and $j_{1}, \ldots, j_{r}$ satisfying the conditions $m_{1} j_{1}+\ldots+m_{r} j_{r}=2 m, j_{1}+\ldots+j_{r}=j$, equals to the best constant the analogue of inequality (1) for nonnegative r.v.'s (see Ibragimov and Sharakhmetov, 2001b). Similar to Remark 1, the latter expression equals to the total number of partitions of a set consisting of $2 m$ elements (the $2 m$-th Bell number).

Let us formulate some auxiliary results needed for the proof of Theorem 1 . The following lemma follows from Corollary 2 in Utev (1985) and the formula representing moments by semi invariants.

Lemma 1. Let $\xi_{1}, \ldots, \xi_{n}$ be independent r.v.'s with $E \xi_{i}^{2 s-1}=0, s=1,2, \ldots, l$. Set $A_{k, n}=\sum_{i=1}^{n} E \xi_{i}^{k}, k=1,2, \ldots, 2 m, B_{n}=A_{2, n}^{1 / 2}$. The following inequality holds:

$$
E\left(\sum_{i=1}^{n} \xi_{i}\right)^{2 m} \leq(2 m) ! \sum_{r=0}^{2 m} \sum \prod_{k=1}^{r} \frac{A_{m_{k}, n}^{j_{k}}\left(m_{k} !\right)^{-j_{k}}}{j_{k} !}
$$


where the inner sum is taken over all natural $m_{1}>m_{2}>\ldots>m_{r}$ and $j_{1}, \ldots, j_{r}$, satisfying the conditions $m_{1} j_{1}+\ldots+m_{r} j_{r}=2 m, j_{1}+\ldots+j_{r}=j, m_{i} \neq 2 s-1, i=1,2, \ldots, r, s=1,2, \ldots, l$.

Let $A_{2 m}, B, D>0$. Denote $M_{1}^{l}\left(m, A_{2 m}, B\right)=\sup E S_{n}^{2 m}$, where sup is taken over $n, \xi_{k}$

positive integers $n$ and all independent r.v.'s $\xi_{1}, \ldots, \xi_{n}$ with $E \xi_{i}^{2 s-1}=0, s=1,2, \ldots, l$ and fixed $A_{2 m, n}=A_{2 m}, B_{n}=B ; M_{2}^{l}\left(m, A_{2 m}, B\right)=\sup _{n, \xi_{k}} E S_{n}^{2 m}$, where sup is taken over positive integers $n$ and all independent r.v.'s $\xi_{1}, \ldots, \xi_{n}$ with $E \xi_{i}^{2 s-1}=0, s=1,2, \ldots, l$, for which $A_{2 m, n} \leq A_{2 m}, B_{n} \leq B$; $M^{l}(m, D)=\sup E S_{n}^{2 m}$, where sup is taken over positive integers $n$ and all independent r.v.'s $n, \xi_{k}$

$\xi_{1}, \ldots, \xi_{n}$ with $E \xi_{i}^{2 s-1}=0, s=1,2, \ldots, l$, and fixed $\max \left(A_{2 m, n}, B_{n}^{2 m}\right)=D$.

The following lemma is well-known (see, e.g., Pinelis and Utev, 1984).

Lemma 2. For $2<s<2 m$

$$
\left|A_{s, n}\right| \leq\left(A_{2 m, n}^{s-2} B_{n}^{2(2 m-s)}\right)^{1 /(2 m-2)} .
$$

Relation (5) and Lemma 2 imply the following

Lemma 3. For $A_{2 m}, B>0$ 


$$
M_{i}^{l}\left(m, A_{2 m}, B\right) \leq(2 m) ! \sum_{j=1}^{2 m}\left(\sum_{r=1}^{j} \sum \prod_{k=1}^{r} \frac{\left(m_{k} !\right)^{-j k}}{j_{k} !}\right)\left(A_{2 m}^{m-j} B^{2 m(j-1)}\right)^{1 /(m-1)},
$$

$i=1,2$, where the inner sum is taken over all natural $m_{1}>m_{2}>\ldots>m_{r}$ and $j_{1}, \ldots, j_{r}$, satisfying the conditions $m_{1} j_{1}+\ldots+m_{r} j_{r}=2 m, j_{1}+\ldots+j_{r}=j, m_{i} \neq 2 s-1, i=1,2, \ldots, r, s=1,2, \ldots, l$.

Proof of Theorem 1. From Lemma 3 and the evident inequality

$$
M^{l}(m, D) \leq M_{2}^{l}\left(m, D, D^{1 / 2 m}\right)
$$

it follows that

$$
M^{l}(m, D) \leq(2 m) ! \sum_{j=1}^{2 m} \sum_{r=1}^{j} \sum \prod_{k=1}^{r} \frac{\left(m_{k} !\right)^{-j_{k}} D}{j_{k} !}
$$

where the inner sum is taken over all natural $m_{1}>m_{2}>\ldots>m_{r}$ and $j_{1}, \ldots, j_{r}$, satisfying the conditions

$$
\begin{gathered}
m_{1} j_{1}+\ldots+m_{r} j_{r}=2 m, j_{1}+\ldots+j_{r}=j, m_{i} \neq 2 s-1, i=1,2, \ldots, r, s=1,2, \ldots, l \text {. Since } \\
C_{l}^{*}(2 m)=\sup _{D>0} \frac{M^{l}(m, D)}{D}
\end{gathered}
$$

this implies (2). 


\section{REFERENCES}

Bestsennaya, E. V. and Utev, S. A. (1991). An exact upper bound for the even moment of sums of independent random variables. Siberian Math. J. 32, 139-141.

de la Peña, V. H. and Giné, E. (1999). Decoupling. From dependence to independence. Randomly stopped processes. U-statistics and processes. Martingales and beyond. Probability and its Applications (New York). Springer-Verlag, New York.

de la Peña, V. H., Ibragimov, R. and Sharakhmetov, S. (2003). On extremal distributions and sharp $L_{p}$-bounds for sums of multilinear forms. Ann. Probab. 31, 630-675.

Figiel, T., Hitczenko, P., Johnson, W. B., Schechtman, G. and Zinn, J. (1997). Extremal properties of Rademacher functions with applications to the Khintchine and Rosenthal inequalities. Trans. Amer. Math. Soc., 349, 997-1027.

Hitczenko, P. (1990). Best constants in martingale version of Rosenthal's inequality. Ann. Probab. 18, 1656-1668.

Hitczenko, P. (1994). On a domination of sums of random variables by sums of conditionally independent ones. Ann. Probab. 22, 453-468.

Ibragimov, R. (1997). Estimates for the moments of symmetric statistics. Ph.D. Dissertation. Institute of Mathematics of Uzbek Academy of Sciences, Tashkent, 127 pp. (in Russian).

Ibragimov, R. and Sharakhmetov, Sh. (1995). On the best constant in Rosenthal's inequality. In: Theses of reports of the conference on probability theory and mathematical statistics dedicated to the 75th anniversary of Academician S. Kh. Sirajdinov (Fergana, Uzbekistan). Tashkent, 43-44 (in Russian). 
Ibragimov, R. and Sharakhmetov, Sh. (1997). On an exact constant for the Rosenthal inequality. Teor. Veroyatnost. i Primen. 42, 341-350 (translation in Theory Probab. Appl. 42 (1997), 294-302 (1998)).

Ibragimov, R. and Sharakhmetov, S. (2001a). The exact constant in the Rosenthal inequality for random variables with mean zero. Theory Probab. Appl. 46, 127-132.

Ibragimov, R. and Sharakhmetov, S. (2001b). The best constant in the Rosenthal inequality for nonnegative random variables. Statist. Probab. Lett. 55, 367-376.

Johnson, W. B., Schechtman, G. and Zinn, J. (1985). Best constants in moment inequalities for linear combinations of independent and exchangeable random variables. Ann. Probab. 13, 234253.

Nagaev, S. V. and Pinelis, I. F. (1977). Some inequalities for the distributions of sums of independent random variables. Theory of Probab. Appl. 22, 248-256.

Pinelis, I. F. (1980). Estimates for moments of infinite-dimensional martingales. Math. Notes 27, 459-462.

Pinelis, I. (1994). Optimum bounds for the distributions of martingales in Banach spaces. Ann. Probab. 22, 1679--1706.

Pinelis, I. F. and Utev, S. A. (1984). Estimates of moments of sums of independent random variables. Theory Probab. Appl. 29, 574-577.

Prokhorov, Yu. V. (1962). Extremal problems in limit theorems. In Proc. VI All-Union Conference on Probability Theory and Mathematical Statistics, Vilnius, 77-84.

Rosenthal, H. P. (1970). On the subspaces of $L^{p} \quad(p>2)$ spanned by sequences of independent random variables. Israel J. Math. 8, 273-303.

Sachkov, V. N. (1966). Combinatorial methods in discrete mathematics. Encyclopedia of Mathematics and its Applications, 55. Cambridge University Press, Cambridge, 306 pp. 
Sazonov, V. V. (1974). On the estimation of moments of sums of independent random variables. Theory Probab. Appl. 19, 371-374.

Utev, S. A. (1985). Extremal problems in moment inequalities. In Proc. Mathematical Institute of the Siberian Branch of the USSR Academy of Sciences, 5, 56-75 (in Russian). 